\title{
Estimation de la conductivité thermique d'un sol in situ, à l'aide d'une sonde à choc thermique
}

\author{
F Bussière 1, P Cellier 2, A Dorigny 3 \\ 1 INRA, unité de bioclimatologie, CRAAG, BP 1232, 97185 Pointe-à-Pitre Cedex; \\ 2 INRA, station de bioclimatologie, 78850 Thiverval-Grignon; \\ ${ }^{3}$ INRA SESCPF, Ardon, 45160 Olivet, France
}

(Reçu le 27 décembre 1990; accepté le 25 novembre 1991)

\begin{abstract}
Résumé - Un dispositif autonome et rapide de mesure in situ de la conductivité thermique des sols, utilisant une sonde à choc thermique monotige a été réalisé. Les conditions d'utilisation et le domaine de validité de la méthode ont été déterminés. D'après des essais effectués sur un sol argilo-limoneux, la conductivité thermique croît avec l'humidité pondérale et la densité pour des sols travaillés. Dans des horizons plus profonds, de forte densité, les valeurs de conductivité thermique restent élevées, même à de faibles humidités, ce qui pourrait résulter de la prise en masse du sol consécutive à son dessèchement. Les valeurs de conductivité thermique que nous avons mesurées sont, pour des humidités élevées, supérieures à celles trouvées par des méthodes de mesure en laboratoire utilisant des sols reconstitués. L'absence de méthode de référence dans ce domaine ne nous a pas permis une validation complète.
\end{abstract}

sol / conductivité thermique / in situ / sonde à choc thermique / méthode de mesure

Summary - Estimating thermal conductivity of soil in situ, with a new thermal probe. A simple system, using a thermal probe was designed to measure the thermal conductivity of a soil in situ. The operating conditions were investigated. The system was shown to be quite adapted for the field experiments. Measurements were made in a siltyclay-loam soil for different humidity and density conditions. As expected, the thermal conductivity increased with gravimetric water content and density of the soil. In the deeper layers, with homogeneous and larger densities, the thermal conductivity had high, steady values even at low water content, which may be due to the shrinkage of clay as the soil dries, compensating the diminution of thermal conductivity due to water losses. This behavior was quite different from that of the tilled surface layers with a granular structure. The measured values of thermal conductivity were higher than those found by laboratory methods using reconstituted soil samples. The absence of a reference method for in situ determination of the thermal conductivity did not allow us to completely validate this thermal probe.

soil / thermal conductivity / in situ / thermal probe / measuring method

\section{INTRODUCTION}

Les transferts de chaleur dans le sol intéressent de nombreux domaines, soit de recherche, soit d'application :

- la modélisation des échanges sol-végétationatmosphère : le flux de chaleur dans un sol nu, $G$, peut représenter plus de $30 \%$ du rayonnement net, $R n$, à l'échelle horaire en cours de journée (Fuchs et Hadas, 1972; Idso et al, 1975). Par nuit claire, le rapport $G / R n$ peut dépasser $70 \%$, ce qui impose une bonne prise en compte des échanges dans le sol lorsque l'on s'intéresse, par exemple, aux températures minimales nocturnes (Cellier et Itier, 1984; Cellier, 1992);

- l'étude des problèmes de réchauffement du sol au printemps pour les estimations de vitesse de levée, de dates de reprise (Buchan, 1982; Morlat, 1989) ou de refroidissement à l'automne.

La simple estimation d'un bilan d'énergie local demande la connaissance des caractéristiques thermiques du sol : conductivité thermique et capacité calorifique. Ces caractéristiques varient fortement selon le sol considéré, soit dans le temps (travail du sol, dessèchement), soit dans 
l'espace (différences entre couches travaillées et non travaillées, profil d'humidité, types de sol), les causes essentielles de variations étant la porosité et l'humidité du sol (de Vries, 1963).

La capacité calorifique peut être estimée de façon satisfaisante, soit par mesure directe calorimétrique, soit par calcul, si l'on connaît les fractions volumiques de chacune des phases : solide (matières minérale et organique), eau, air (de Vries, 1963). II n'en est par contre pas de même pour l'estimation de la conductivité thermique, pour laquelle aucune méthode de référence n'existe actuellement (Balabanis, 1987). Celle-ci ne dépend pas, en effet, seulement de la conductivité de chacune des phases (eau : 0,6; air : 0,037 ; fraction solide : 3,0 à $8,5 \mathrm{~W} \cdot \mathrm{m}^{-1} \cdot \mathrm{K}^{-1}$ ) mais également de la qualité des contacts entre les éléments (de Vries, 1963).

Nous présentons ici les résultats préliminaires obtenus à l'aide d'une sonde à choc thermique de conception nouvelle (Laurent, 1986) développée au CSTB (Centre scientifique et technique du bâtiment) de Saint-Martin-d'Hères (Isère) (brevet d'invention $n^{\circ} 85$ 19137) et conçue pour estimer directement la conductivité thermique de matériaux de bâtiment, et dont nous avons voulu tester les performances sur un sol naturel.

\section{PROBLÈMES LIÉS À L'ESTIMATION DE LA CONDUCTIVITÉ THERMIQUE D'UN SOL}

\section{Conductivité thermique apparente}

Dans un sol, milieu poreux partiellement saturé, les transferts de chaleur et d'humidité sont étroitement couplés. Les transferts thermiques ne sont pas purement conductifs, en raison notamment des mouvements de chaleur liés aux transferts de vapeur d'eau. Toutefois, ces derniers ne sont réellement significatifs que dans les couches les plus superficielles du sol, en cas d'évaporation intense (de Vries et Philip, 1986), et en règle générale on peut se ramener à une expression classique de la loi de Fourier :

$$
C_{\star} \mathrm{d} T / \mathrm{d} t=\operatorname{div}\left(\lambda_{\star} \operatorname{grad}(T)\right)
$$

où $\lambda_{*}$, conductivité thermique, et $C_{*}$, capacité calorifique, sont les paramètres thermiques apparents du milieu, prenant implicitement en compte les éventuels transferts de chaleur liés aux transferts de vapeur d'eau. Ce sont ces paramètres thermiques apparents auxquels donnent accès l'ensemble des techniques d'estimation de la conductivité thermique d'un sol.

\section{Méthodes d'estimation de la conductivité thermique d'un sol in situ}

Si l'on excepte les méthodes analytiques et empiriques (de Vries, 1963; Mac Innes, 1981) dont les résultats sont assez controversés, et le domaine de validité souvent mal défini (Asrar et Kanemasu, 1983; Balabanis, 1987), les méthodes d'estimation de la conductivité thermique in situ peuvent se classer en 2 grandes catégories :

- les méthodes fondées sur la résolution de l'équation de la chaleur unidimensionnelle, qui utilisent des valeurs de températures mesurées à différents niveaux dans le sol, en conditions naturelles; certains niveaux tiennent lieu de signal d'entrée, alors que d'autres servent au calage de la variable recherchée. Parmi les nombreuses méthodes existantes, Horton et al (1983) ont montré que la méthode harmonique et les méthodes numériques étaient les plus fiables. En outre, Asrar et Kanemasu (1983) ont montré l'intérêt de l'utilisation de la transformée de Laplace.

Une fois les sondes de température installées dans le sol, ces méthodes permettent une estimation de la conductivité ou de la diffusivité thermique dans une grande variété de conditions (dans le temps, et selon la profondeur). Elles présentent l'intérêt de faire relativement peu d'hypothèses sur les conditions de transferts thermiques.

Elles présentent cependant l'inconvénient de nécessiter des calculs assez complexes (au moins pour les 3 méthodes les plus fiables évoquées ci-dessus) et de nombreuses données réparties sur des intervalles de temps longs : 12 à 24 heures (Richards et al, 1989).

- les méthodes à choc thermique qui imposent un apport d'énergie localisé au sol, à l'aide d'une source de chaleur artificielle, dont on analyse la transmission dans le sol soit en géométrie plane à partir de source plane (Van Wijk et al, 1967), soit plus généralement en géométrie cylindrique à partir d'une source linéaire (de Vries et Peck, 1958a; Carslaw et Jaeger, 1959; Fritton et al, 1974; Achard et al, 1984). Laurent (1986) a effectué une revue des différentes sondes à choc thermique de ce dernier type (monotige, bitige et tritige) où il présente les caractéristiques thermiques qu'elles permettent d'obtenir (conductivité 
ou diffusivité) et leurs avantages et inconvénients respectifs. Dans le cas qui nous intéresse, les sondes monotiges présentent le plus d'intérêt car elles sont relativement simples à réaliser, faciles à insérer dans le sol, et le calcul de la conductivité est élémentaire.

\section{MATÉRIEL ET MÉTHODES}

\section{La sonde à choc thermique monotige}

Une sonde à choc thermique est constituée de 2 parties (fig 1):

- un élément cylindrique résistif dans lequel est dissipée, par effet Joule, une quantité de chaleur constante pendant la durée de la mesure;

- une sonde de température placée à l'interface solsonde, au milieu de l'élément chauffant.

La température mesurée tout au long de la durée de chauffage sera le signal de sortie à partir duquel pourra être déterminée la conductivité thermique du milieu (fig 2a).

Moyennant les hypothèses suivantes:

$\mathrm{H}_{1}$ : sol homogène, isotrope et infini;

$\mathrm{H}_{2}$ : température du sol uniforme et stable avant chauffage,

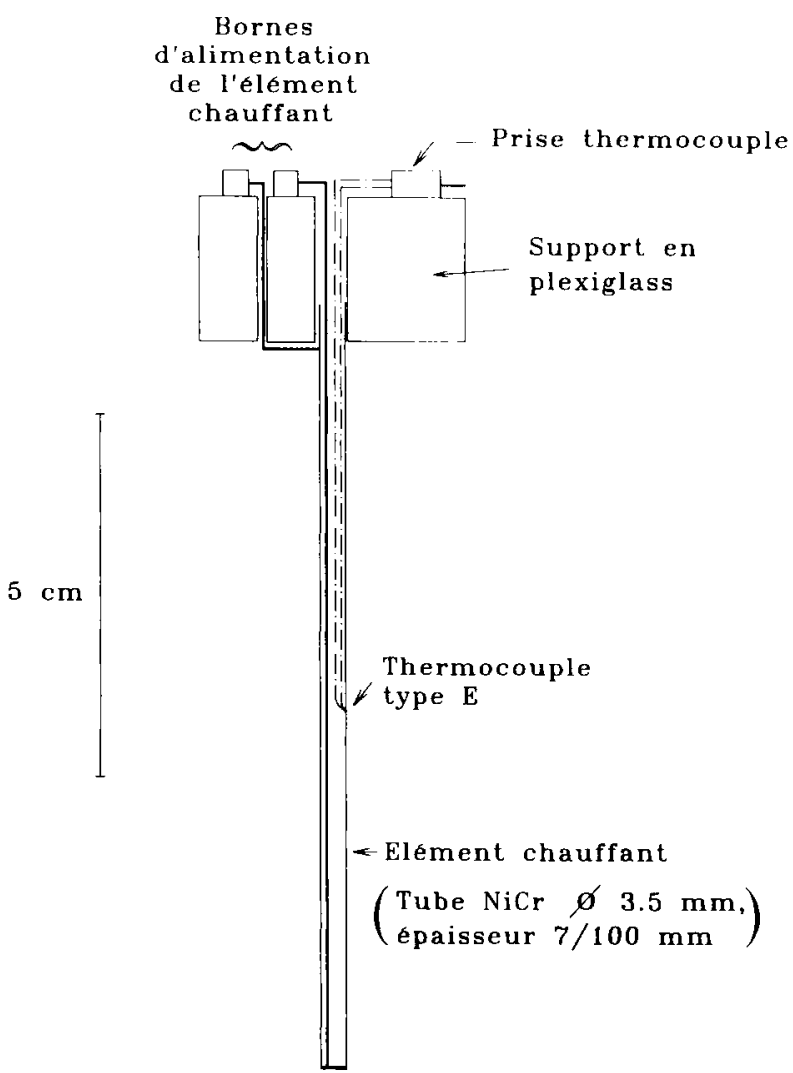

Fig 1. Description d'une sonde à choc thermique monotige : élément chauffant et sonde de mesure de temperature.
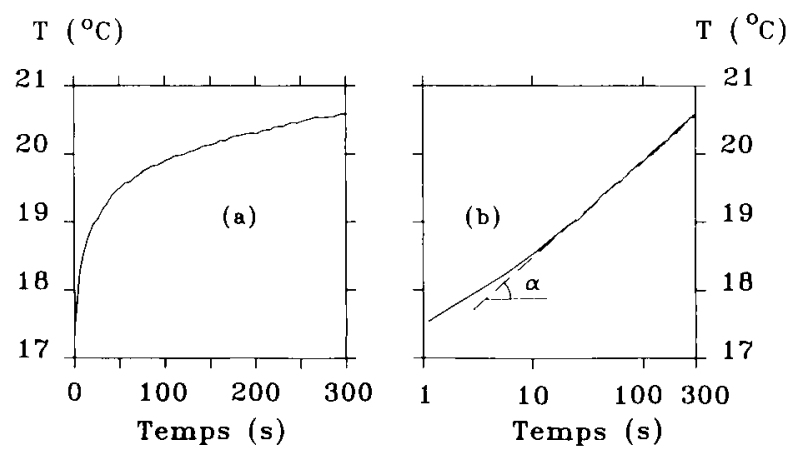

Fig 2. Cinétique de température au cours d'un cycle de mesure à la sonde à choc thermique : (a) en coordonnées normales; (b) en coordonnées semi-logarithmiques. La droite en tirets a été ajustée sur les mesures effectuées entre 180 el $300 \mathrm{~s}$ après le début du chauffage. Sa pente $\alpha=Q_{\theta} /\left(4 \pi \lambda_{*}\right)$ permet de calculer la conductivité thermique.

la résolution de l'équation de la chaleur en géométrie cylindrique dans le système sonde-sol aboutit à la solution asymptotique suivante (Laurent, 1986) :

$\lambda_{*} \Delta T / Q_{\theta}=1 / 4 \pi\left(\ln (t)+\ln \left(4 a / D_{\theta} / R_{s}^{2}\right)+2 . \lambda_{*} \cdot R_{C} / R_{s}\right)(2)$

$\Delta T=$ différence de température $\left({ }^{\circ} \mathrm{C}\right)$ de la sonde entre l'instant $t$ et l'instant initial,

$Q_{e}=$ flux électrique de chauffage injecté par unité de longueur $\left(\mathrm{W} \cdot \mathrm{m}^{-1}\right)$,

$t=$ temps écoulé depuis le début du chauffage (s),

$a=$ diffusivité thermique du matériau $\left(a=\lambda_{\star} / C_{\star}\right)$ $\left(m^{2} \cdot s^{-1}\right)$,

$D_{e}=$ constante (s),

$R_{s}=$ rayon de la sonde $(\mathrm{m})$,

$R_{c}=$ résistance de contact sol-sonde $\left(\mathrm{m}^{2} \cdot \mathrm{K}^{-1} \cdot \mathrm{W}^{-1}\right)$.

La conductivité est alors obtenue de manière simple en calculant la pente $\alpha=Q_{e} /\left(4 \pi \lambda_{*}\right)$ de la régression linéaire entre $\Delta T$ et $\ln (t)$ (fig $2 b$ ) comme le propose Laurent (1986). La méthode qui consisterait à ne retenir que 2 points de mesure n'est pas recommandée en raison de l'incertitude induite sur l'estimation de $\lambda$ (de Vries et Peck, 1958a, b).

Ce formalisme suppose que la sonde soit suffisamment longue pour que les lignes de flux puissent être considérées comme radiales (Laurent, 1986).

\section{Problèmes liés aux mesures in situ sur un sol naturel avec une sonde à choc thermique}

En milieu naturel apparaissent de nombreuses contraintes, d'une part d'ordre technique, d'autre part liées au milieu étudié, le sol, qui vont invalider partiellement les hypothèses faites.

D'un point de vue physique, le sol est un milieu hétérogène à l'échelle du $\mathrm{cm}$, tant par sa nature (sols plus ou moins caillouteux), que par les travaux culturaux dont il a été l'objet (labour, lit de semence). II est, 
de ce fait, nécessaire d'utiliser une sonde de dimensions suffisantes afin d'intégrer cette hétérogénéité locale. En outre, avant de pouvoir affirmer que l'hypothèse $\mathrm{H}_{1}$ est vérifiée, il est nécessaire d'avoir une estimation du volume d'influence de la sonde. On peut en obtenir une approximation en utilisant l'équation suivante qui donne la variation de température $\Delta T_{r}$ à une distance $r$ d'une source linéaire de chaleur (Carslaw et Jaeger, 1959) :

$$
\Delta T_{r}=Q_{\theta} / 4 \pi \lambda \cdot \exp \left(-r^{2} / 4 a t\right)
$$

II apparaît clairement que le volume d'influence dépend des caractéristiques thermiques elles-mêmes, et évolue dans le sens inverse de celles-ci. En considérant comme volume d'influence la zone où l'élévation de température est supérieure à $10 \%$ de l'accroissement de température de la sonde, on obtient un rayon d'influence de l'ordre de $20 \mathrm{~mm}$ pour des caractéristiques thermiques moyennes. II est donc relativement aisé de choisir un lieu d'insertion de la sonde qui satisfasse l'hypothèse $\mathrm{H}_{1}$ dans un sol naturel.

L'hypothèse $\mathrm{H}_{2}$ est également difficile à satisfaire, puisque le sol est l'objet de variations très importantes de température dans le temps ou l'espace (gradients verticaux, notamment). II est possible de contourner cette difficulté en utilisant, non pas directement la température de la sonde, mais plutôt son écart à la température du sol non chauffé, ce qui permet, d'après Jury et Bellantuoni (1976) d'améliorer la mesure en intégrant la variation naturelle de température du sol.

Un problème important posé par ce type de sonde est la qualité du contact entre la sonde et le matériau qui ne permet pas toujours d'atteindre la solution asymptotique (équation (2)) lorsque la résistance de contact est trop forte (Hadas, 1974; Laurent, 1986). Ce problème est particulièrement sensible dans un matériau meuble comme un sol, surtout dans les couches les plus superficielles (lit de semence). À ce titre, la sonde que nous avons utilisée s'avérait d'une conception particulièrement intéressante, sa très faible inertie thermique permettant de s'approcher plus rapidement du régime asymptotique (Laurent, 1986).

L'un des objectifs de ce travail consistait à juger de la possibilité d'utiliser une sonde à choc thermique lorsqu'on se trouve à la limite de ses conditions d'emploi.

\section{Description de la sonde utilisée et du système de mesures associé}

L'élément chauffant de la sonde est constitué d'un tube en alliage de nickel et chrome de $100 \mathrm{~mm}$ de longueur, de diamètre $3,5 \mathrm{~mm}$, d'épaisseur de paroi 0,07 $\mathrm{mm}$ et de masse linéique égale à $6 \mathrm{~g} / \mathrm{m}$; la résistance de cet élément est de 0,15 à $0,16 \Omega$. Le courant qui traverse la sonde provient d'une batterie au plomb de $12 \mathrm{~V}$, et il est contrôlé très précisément (variations inférieures à $1 \mathrm{~mA}$ ) par un régulateur de conception simple réalisé au laboratoire, permettant de générer un courant variant entre 2,5 et $4 \mathrm{~A}$. Le choix de la puissance dissipée (donc de la valeur du courant imposé) résulte d'un compromis entre le besoin d'obtenir une augmentation substantielle de température, permettant une bonne estimation de la pente en régime asymptotique (formule (2)), mais restant dans des limites évitant la création de gradients thermiques suffisants pour induire des mouvements notables d'eau. Pour tous les résultats présentés ici, le courant de $3 \mathrm{~A}$ provoquait des augmentations de température de 3 à $5^{\circ} \mathrm{C}$ dans la quasi-totalité des cas, mais pouvant aller jusqu'à plus de $10^{\circ} \mathrm{C}$ dans les couches de surface les plus sèches (humidité pondérale inférieure à 10\%).

L'ensemble du système était piloté par une centrale de données Campbell Scientific CR10 fonctionnant sur batterie qui réalisait les fonctions suivantes :

- contrôle de stabilité de la température de la sonde : jugée stable quand 3 mesures successives $(5 \mathrm{~s}$ entre 2 mesures) sont identiques (au 1/100 de K);

- déclenchement du chauffage;

- mesure de la température de la sonde et du sol voisin toutes les $5 \mathrm{~s}$;

- arrêt au bout de $300 \mathrm{~s}$;

- calcul de la conductivité sur les 3 dernières min;

- stockage de l'ensemble des données (heure, températures de la sonde et du sol non chauffé, courant), et des calculs effectués pour contrôle éventuel.

\section{Dispositif expérimental}

Afin d'obtenir une gamme de conductivite thermique aussi large que possible, nous avons effectué des mesures pour un sol limono-argileux à Grignon (France, latitude $48,51^{\circ} \mathrm{N}$, longitude $1,58^{\circ} \mathrm{E}$ ), dans des conditions d'humidité et de densité du sol variables.

Dans la suite du texte, chacun des traitements sera repéré par un nombre à 2 chiffres :

- le chiffre des unités identifie l'horizon cultural (un niveau de mesure par horizon, dont l'épaisseur dépend du tassement) : 1 = lit de semence (profondeur : 3 ou $5 \mathrm{~cm}$ selon que le sol est tassé ou non), 2 = zone labourée (profondeur : 15 ou $12 \mathrm{~cm}$ ), $3=$ sol profond (profondeur : $35 \mathrm{~cm}$ );

- le chiffre des dizaines correspond aux divers traitements effectués, comme suit :

- 4 préparations du sol différentes, dont les 3 premières correspondent à des situations agronomiques classiques : labour effectué à l'automone non repris $(1 x)$, lit de semence grossier $(2 x)$, lit de semence fin $(3 x, 5 x$ et $7 x$ selon l'état de sécheresse : voir cidessous); enfin un sol compacté $(4 x, 6 x$, ou $8 x$ selon l'état de sécheresse);

- des traitements humides obtenus par un arrosage de complément de $50 \mathrm{~mm}$ (traitements $1 x, 2 x, 3 x$ et $4 x$ ); les observations y ont été effectuées les 15 et 16 juin 1988 pour les traitements $1 x, 2 x$ et $3 x$ et le 29 juin 1988 pour le $4 x$; 
- des traitements secs placés sous un tunnel plastique de $6 \mathrm{~m} \times 6 \mathrm{~m}$; le tunnel évitait en outre une trop forte dégradation des couches superficielles du sol, et donc une évolution importante de leur densité; des observations ont été effectuées à 2 étapes de dessèchement : $5 x$ et $6 x$ le 27 juillet 1988 , et $7 x$ et $8 x$ le 25 août 1988 .

\section{Protocole expérimental}

Juste après l'arrosage des traitements humides, la surface du sol était recouverte d'une feuille plastique réfléchissante, afin d'homogénéiser la température et l'humidité du milieu, jusqu'au moment des mesures. Sur l'ensemble des traitements, une fosse était creusée juste avant chaque série d'observations, et la surface du sol recouverte d'une plaque de polystyrène expansé, permettant de limiter les variations brutales de température (d'origine météorologique) pendant la durée des mesures dans les couches superficielles.

La sonde était insérée horizontalement dans le sol en réalisant un prétrou à l'aide d'une mèche à fort dégagement montée sur une perceuse fonctionnant sur batterie $(300 \mathrm{t} / \mathrm{min})$ et maintenue stable à l'aide d'un guide.

À la suite de chaque mesure, après le retrait de la sonde, un cylindre de sol de $15 \mathrm{~mm}$ de rayon, axé sur l'emplacement de la sonde, était prélevé pour mesure d'humidité pondérale et de densité par pesée.

\section{RÉSULTATS}

En l'absence de comparaison avec une méthode de référence, les résultats obtenus présentent un caractère tout à fait préliminaire traduisant surtout les performances techniques de cette sonde. Nous présentons également une illustration de l'évolution de la conductivité thermique ainsi obtenue avec les caractéristiques du sol les plus susceptibles de l'affecter : humidité et densité.

\section{Performances techniques}

Le système présenté ci-dessus répond tout à fait aux besoins de l'estimation in situ de la conductivité thermique :

- léger et facile à mettre en place dans une large gamme de sols : lit de semence, sol très tassé et même sol caillouteux;

- autonome : avec une batterie au plomb courante (12 V, $40 \mathrm{Ah}$ ), il est possible d'effectuer une centaine de mesures;

- permettant une estimation rapide : la valeur de conductivité est disponible dès la fin de la me- sure (une dizaine de min s'écoulent entre l'insertion et le retrait de la sonde).

\section{Qualité de la mesure}

L'ensemble des essais a permis de mettre en évidence les faits suivants :

- une bonne reproductibilité : des mesures effectuées successivement au même emplacement toutes les $4 \mathrm{~h}$ (afin de permettre à la température du sol de se réhomogénéiser) nous ont donné une valeur moyenne de $1,25 \mathrm{~W} \cdot \mathrm{m}^{-1} . \mathrm{K}^{-1}$ avec un écart type de 0,05 (12 valeurs). En outre, on peut voir sur le tableau I que l'ensemble des valeurs mesurées dans l'horizon le plus profond (le plus homogène en densité et humidité) sont très peu dispersées;

- le temps de chauffage de 5 min est tout à fait suffisant. Sur la figure 3 , on a porté les valeurs de conductivités calculées sur différents intervalles de temps en fonction de $\lambda_{\text {ref }}$, la valeur calculée à partir des mesures effectuées entre 180 et $300 \mathrm{~s}$ (période située bien au-delà du début de la phase asymptotique). On voit que la valeur obtenue est stable dès $60 \mathrm{~s}$ après le début du chauffage. La limitation du temps de chauffage permet de réduire l'élévation de température et les transferts de vapeur d'eau qui lui sont associés, qui affectent d'autant plus la mesure de la conductivité thermique que le temps de chauf-
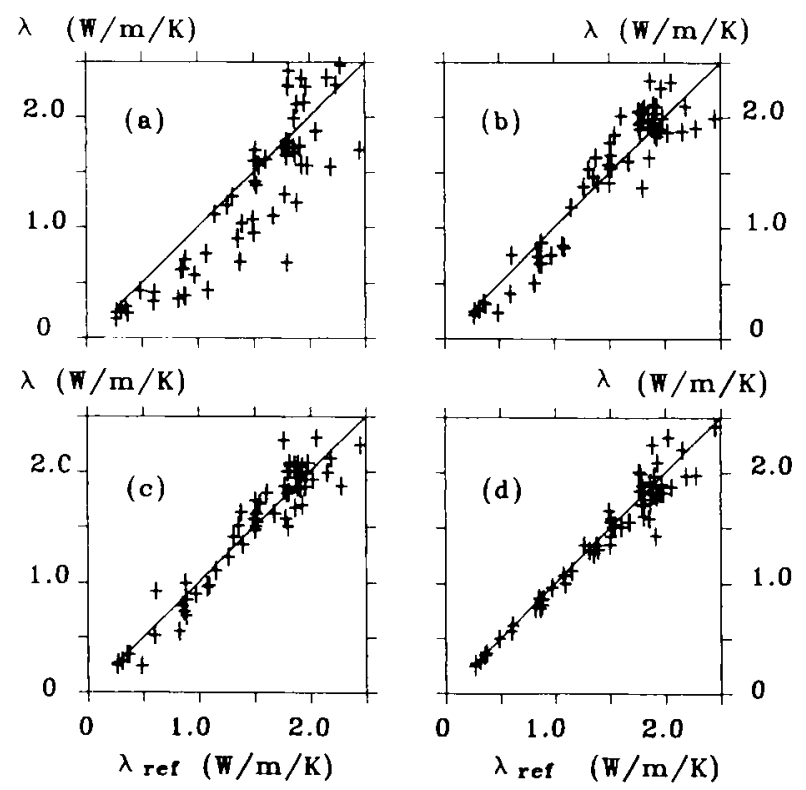

Fig 3. Conductivités thermiques estimées sur différents intervalles de temps (exprimés en s après le début du chauffage) en fonction de $\lambda_{\text {ref }}$, la valeur calculée sur l'intervalle 180-300 s : (a) intervalle 0-60 s; (b) 30-90 s; (c) 60-120 s; (d) $180-240 \mathrm{~s}$. 
Tableau I. Valeurs moyennes, écarts types et nombres de mesures pour les différents paramètres mesurés sur l'ensemble des traitements et pour les 3 horizons (voir texte) : $\lambda$ : conductivité thermique $\left(W \cdot \mathrm{m}^{-1} \cdot \mathrm{K}^{-1}\right) ; D:$ densité du sol; $\Theta_{p}$ : humidité pondérale du sol $(\mathrm{g} / \mathrm{g}) ; \Theta_{v}$ : humidité volumique du sol $\left(\mathrm{m}^{3} / \mathrm{m}^{3}\right)$. Moyenne; $<$ : écart type; ( ): nombre de mesures; / : pas de mesure possible.

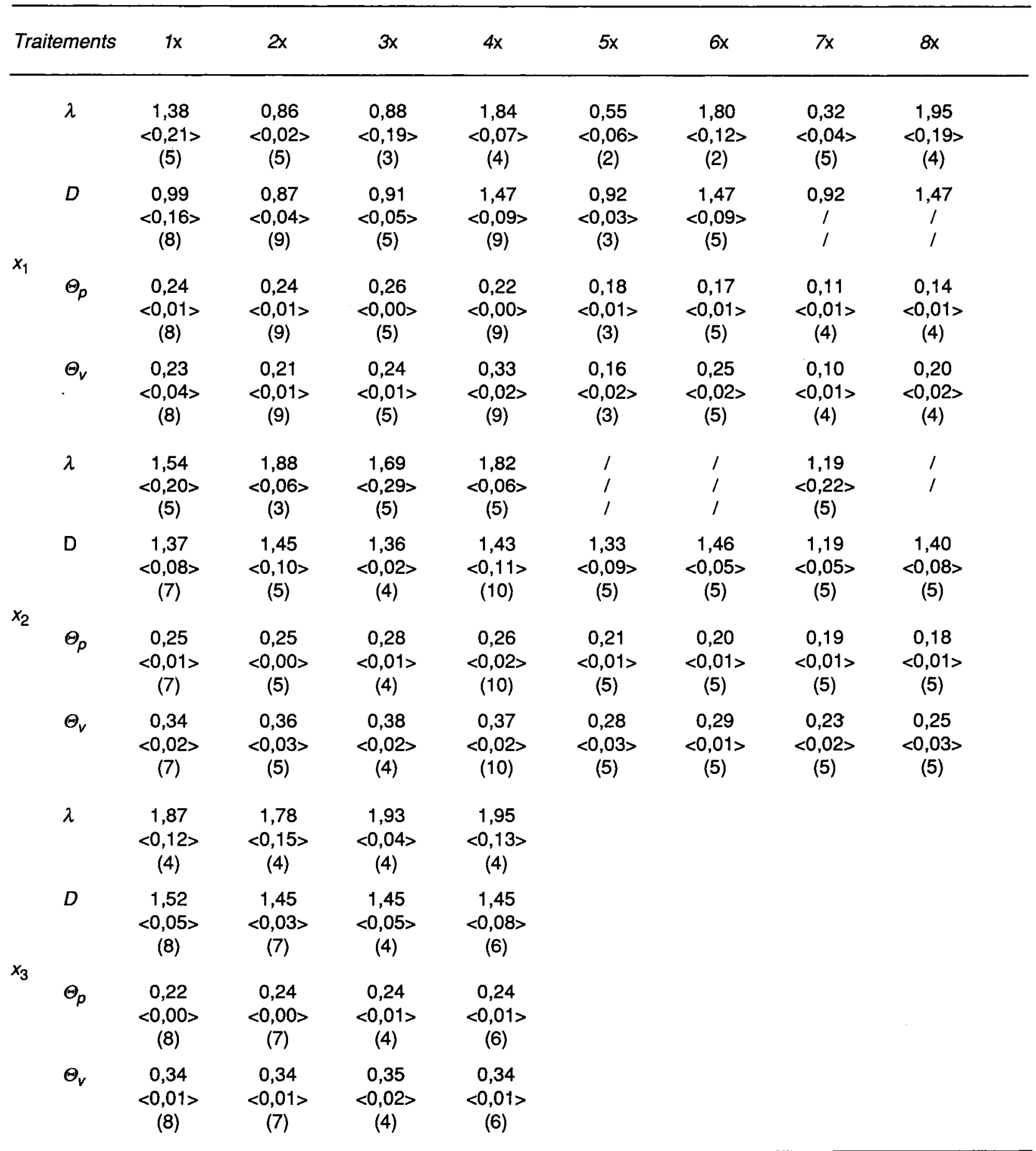

fage est long (de Vries et Peck, 1958b; Hadas, 1974). En outre, en début de chauffage l'ajustement est en règle générale meilleur car la variation de température est alors plus importante (fig 2);

- l'hypothèse d'homogénéité $\left(H_{1}\right)$ est raisonnable pour un sol, puisque même dans le cas d'un sol caillouteux, on obtient pour un même niveau une valeur moyenne de conductivité thermique de $1,01 \mathrm{~W} \cdot \mathrm{m}^{-1} . \mathrm{K}^{-1}$ avec un écart type de 0,04 (11 valeurs).

L'ensemble des résultats est porté sur le tableau I On notera la grande variabilité qui pourrait avoir plusieurs origines : 
- la nature du milieu : traitements 11 et 12 (labour motteux), ainsi que 81 (sol compacté et sec fissuré);

- difficultés d'insertion de la sonde dans le sol : traitement 81 (compacté et sec);

- le faible nombre de mesures, souvent liés à des problèmes techniques : traitements 31 et 32 vraisemblablement.

\section{Relation entre la conductivité et l'humidité pondérale}

Les valeurs moyennes de conductivité pour chaque traitement sont portées sur la figure 4 en fonction de l'humidité pondérale, pour différentes classes de densité. En dehors des points relevés dans l'horizon le plus profond (traitement $\times 3$ ) dans lequel l'humidité varie peu et la conductivité thermique reste très stable, on distingue 2 grands types de comportement dans les différentes catégories de densité :

- d'une part, les couches travaillées (traitements $x 1$ et $x 2$ ). La relation apparaît monotone et croissante. Les mesures effectuées dans les différents horizons se distinguent clairement : les valeurs de conductivité thermique sont plus faibles pour les mesures effectuées dans le lit de semence que dans la zone labourée (traitements $31,51,71$ et $12,32,72$ respectivement);

- d'autre part, le sol plus tassé, profond (x3) ou compacté $(41,61,81)$ pour lequel la relation est

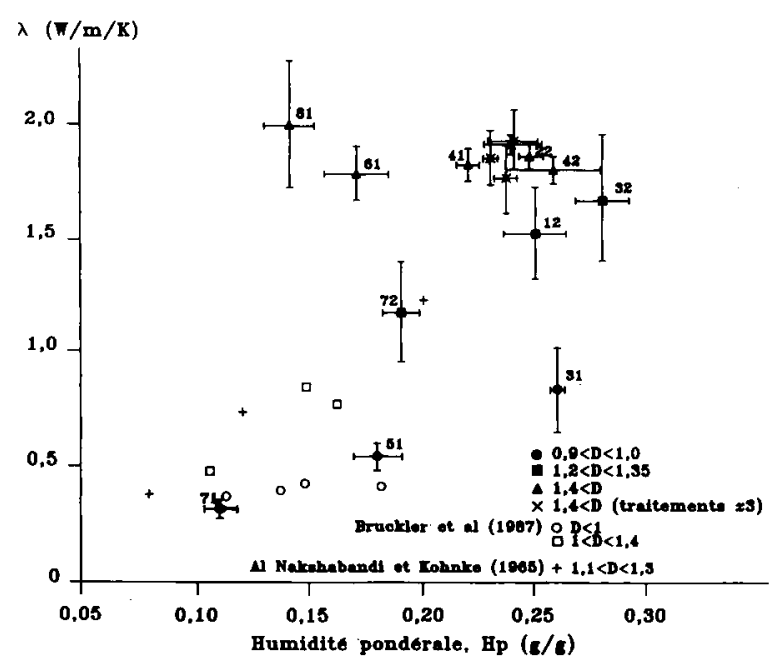

Fig 4. Conductivité thermique du sol en fonction de son humidité pondérale, pour différentes classes de densité; les barres verticales et horizontales représentent les écarts types et le nombre porté à côté du point identifie le traitement (les valeurs sont données au tableau l). beaucoup moins claire, et où l'on n'observe pas de tendance dans l'évolution de la conductivité thermique avec l'humidité pondérale.

Cette différence pourrait résulter des variations de contact entre les éléments minéraux du sol des différents traitements. À partir d'un état structural donné, 2 facteurs peuvent améliorer ce contact : la présence d'eau qui, par la formation de ménisques, augmente fortement les surfaces de contact entre éléments minéraux, et le retrait, notamment dans les sols argileux en cours de dessèchement (de Vries, 1963; Hallaire, 1988). Dans le cas du lit de semence, le retrait ne peut être efficace car il ne s'exerce qu'à l'intérieur des agrégats, et n'améliore donc pas le contact entre agrégats nécessaire à une bonne conduction de la chaleur. On peut ainsi s'attendre à observer une conductivité d'autant meilleure que l'humidité du sol augmente, celleci étant le seul facteur susceptible d'améliorer le contact entre agrégats. Pour les horizons plus denses ( $x 2$ et $4 x$ ), la prise en masse consécutive à un dessèchement important pour un sol ayant une proportion notable d'argile (20\%) (Hallaire, 1988), pourrait compenser, du point de vue de la conductivité thermique, les pertes d'eau.

\section{Relation entre conductivité et densité}

On a porté sur la figure 5 la conductivité thermique en fonction de la densité du sol pour différentes classes d'humidité pondérale. Dans tous les cas, la relation est croissante, et les comportements des différentes classes d'humidité sont proches les uns des autres. La densité traduit indirectement, à la fois le rôle des contacts entre éléments minéraux du sol, ainsi qu'entre agrégats et de sa porosité. Nos mesures montrent l'influence globale de ces différents paramètres sur la conductivité, mais ne permettent pas de discriminer leurs importances relatives. On peut toutefois estimer que, dans notre cas, la relation entre conductivité et densité est fortement liée aux différences de structure entre les horizons, comme nous l'avons souligné au paragraphe précédent.

La forte variabilité de la densité du traitement 11 , ainsi que sa variation relativement forte de conductivité, si on la compare à celle des autres traitements similaires $(21,31,41,51$ et 71$)$ résulte de l'état structural du traitement (labour motteux) dans lequel peuvent exister des poches d'air qui rendent également les mesures de densité délicates. 


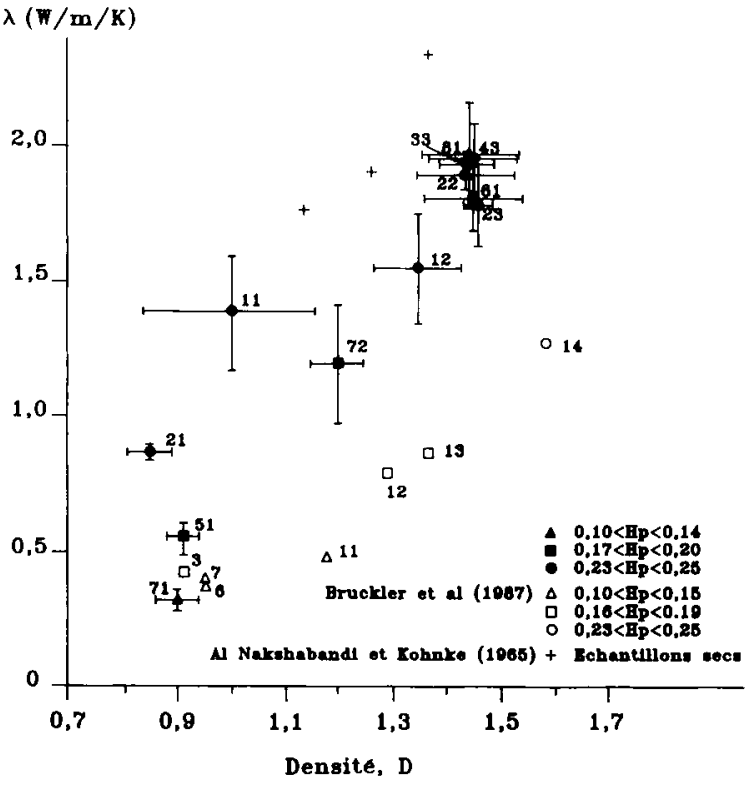

Fig 5. Conductivité thermique du sol en fonction de sa densité, pour différentes classes d'humidité pondérales; mêmes conventions que pour la figure 4 .

\section{Relation entre conductivité et humidité volumique}

L'humidité volumique intègre à la fois des informations sur la densité et sur l'humidité pondérale. On retrouve donc, sur la figure 6 , l'ensemble des informations présentées sur les figures 4 et 5 . On a également porté sur cette figure les résultats expérimentaux de Hopmans et Dane (1986) et Ghuman et Lal (1985), obtenus sur des échantillons remaniés ayant une forte densité ( 1,58 et 1,50 respectivement).

\section{DISCUSSION ET CONCLUSION}

Nous avons pu vérifier le bon fonctionnement de ce type de sonde et son adéquation à l'estimation de la conductivité thermique in situ. En se plaçant dans des conditions de densité et d'humidité bien différenciées, on a pu souligner l'effet prépondérant de la densité du sol, et donc du contact entre éléments minéraux sur la conductivité thermique. Pour des considérations pratiques, ce résultat est intéressant car la densité d'un sol est beaucoup moins susceptible de varier au cours du temps que son humidité. Comme on a vu également que le retrait du sol compensait partiellement la perte d'eau, cela

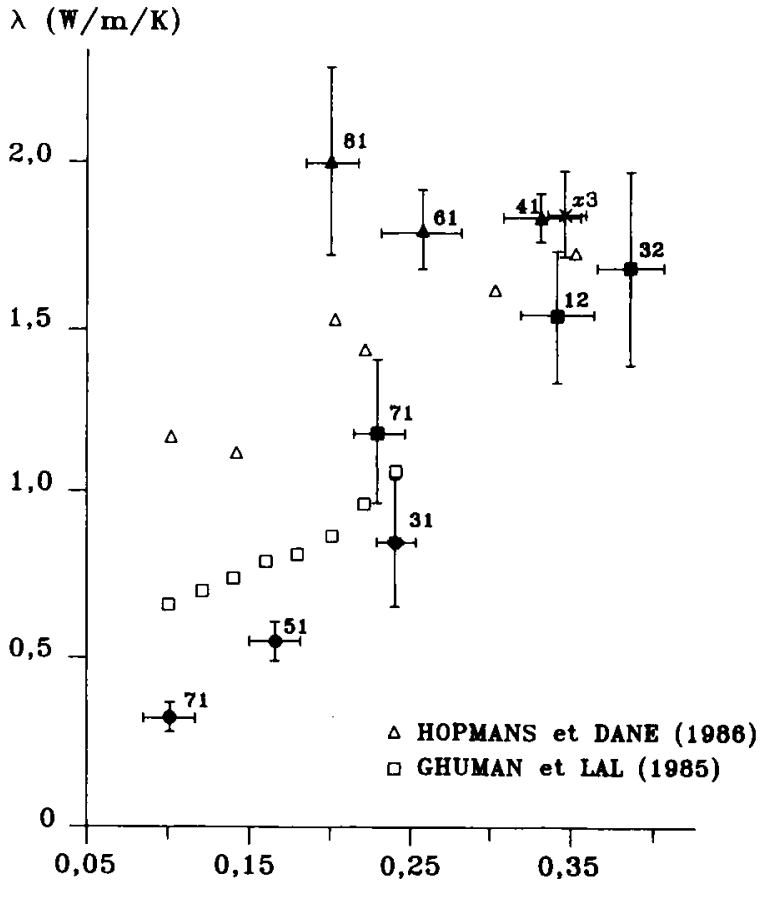

Humidite volumique $\left(\mathrm{m}^{3} / \mathrm{m}^{3}\right)$

Fig 6. Conductivité thermique du sol en fonction de son humidité volumique, pour différentes classes de densité; notations cf figure 4 .

permet de considérer la conductivité comme constante sur une large gamme d'humidités pour les horizons profonds. C'ést essentiellement dans les couches de surface que les caractéristiques thermiques sont susceptible de changer en raison, d'une part, des variations de structure, et d'autre part, des fortes variations d'humidité qu'on peut $y$ attendre.

N'ayant pu valider directement nos résultats à l'aide d'une méthode de référence, nous avons cherché à les comparer à des mesures de conductivité publiées. Ces comparaisons sont toutefois très qualitatives puisqu'elles s'appliquent à des mesures faites dans des conditions différentes.

On peut voir sur la figure 4 que, pour des classes de densités équivalentes, les résultats de Bruckler et al (1987) sont tout à fait comparables aux nôtres pour des humidités faibles : leurs échantillons de densité supérieure à 1,0 s'inscrivant globalement dans la tendance de la deuxième classe de densité de notre étude. La même remarque peut être faite sur l'ensemble de la gamme d'humidité pour les mesures de Al Nakshabandi et Kohnke (1965) qui ont également utilisé une sonde à choc thermique, mais 
avec une procédure de dépouillement des cinétiques de température plus sommaire. La densité du sol limoneux sur lequel ont été obtenues les valeurs de conductivité portées sur la figure 4 est comprise entre 1,15 et 1,30 .

Par contre, la figure 5 montre un net désaccord : si les valeurs estimées par Bruckler et al (1987) à faibles densités sont proches des nôtres, on remarque cependant que l'augmentation de conductivité avec la densité est plus faible pour leurs données. À l'inverse, les valeurs de conductivité estimées par Al Nakshbandi et Kohnke (1965) pour des échantillons secs sont plus élevées que les nôtres, à densité égale, même si les pentes des relations entre conductivité thermique et densité ne semblent pas très éloignées. Ces différences peuvent résulter de difficultés techniques dans les méthodes utilisées:

- difficultés à recréer un sol dans un petit cylindre au laboratoire pour Bruckler et al (1987) et Al Nakshabandi et Kohnke (1965);

- transfert éventuel de vapeur d'eau consécutif à l'existence d'un gradient thermique durable dans le cas de Bruckler et al (1987) (de Vries et Peck, 1958b; Hadas, 1974);

- problèmes de contact entre le sol et la sonde, et également transferts de vapeur dans notre expérimentation; toutefois un mauvais contact entre le sol et la sonde induirait plutôt une sousestimation de la conductivité thermique (Hadas, 1974; Laurent, 1986); en outre, les élévations de températures aux fortes conductivités sont faibles $\left(2-3^{\circ} \mathrm{C}\right)$ et de courte durée, et n'induisent donc, très vraisemblablement, que de faibles transferts de vapeur.

Ces divergences, opposées à la similitude des comportements en ce qui concerne l'évolution de la conductivité avec la densité, soulignent bien la complexité du déterminisme de la conductivité thermique dans laquelle la qualité des contacts entre éléments minéraux joue un rôle important (de Vries, 1963).

Les données de Hopmans et Dane (1986) et Ghuman et Lal (1985) portées sur la figure 6 sont tout à fait comparables aux nôtres, même si la pente de la relation entre la conductivité et l'humidité volumique semble plus faible pour ces derniers que pour nos données. II faut toutefois souligner que ces 2 sols ont des caractéristiques différentes de celles de notre terrain expérimental : densités plus fortes (supérieures à 1,5) et sol utilisé par Hopmans et Dane (1986) plus sableux; or les sols sableux ont, en règle générale, des conductivités thermiques plus élevées que les sols limoneux comme celui que nous avons utilisé.

De manière générale, hormis pour les données de Al Nakshabandi et Kohnke (1965), la sonde à choc thermique donne des valeurs de conductivités thermiques supérieures aux données publiées que nous avons trouvées, au moins aux fortes humidités. II semble toutefois difficile de trancher, en l'absence d'une véritable méthode de référence pour l'estimation de la conductivité thermique in situ. Compte tenu du très bon comportement au champ de la sonde que nous avons utilisée, il semble intéressant de poursuivre cet effort de validation. Plusieurs voies sont possibles : en laboratoire, par comparaison avec d'autres méthodes directes (celle décrite par Brucklert et al (1987), par exemple) ou au champ grâce à des mesures simultanées de température et de teneur en eau, par inversion de modèles numériques.

\section{REMERCIEMENTS}

Nous tenons à remercier ici le Centre scientifique et technique du bâtiment de Saint-Martin d'Hères (Isère) qui nous a gracieusement donné les 5 sondes à choc thermique utilisées dans cette étude.

\section{RÉFÉRENCES}

Achard G, Roux JJ, Sublet JC (1984) Description d'une sonde de mesure des caractéristiques thermiques des couches superficielles du sol. Résultats d'une campagne de mesure. Rev Gén Therm Fr 267, 177-188

Asrar G, Kanemasu ET (1983) Estimating thermal diffusivity near the soil surface using Laplace transform: uniform initial conditions. Soil Sci Soc Am J 47, 397-401

Balabanis P (1987) Caractérisation thermique des sols partiellement saturés. Application à la prédiction in situ du gel des couches de surface. Thèse de l'Université scientifique, technologique et médicale de Grenoble, Grenoble, France, 154 p

Bruckler L, Renault P, Aries F (1987) Laboratory estimation of apparent soil thermal conductivity using a numerical approach. Soil Sci 143, 387-397

Buchan GD (1982) Predicting bare soil temperature II. Experimental testing of multi-day models. J Soil Sci 33, 199-209

Carslaw HS, Jaeger JC (1959) Conduction of heat in solids. Oxford Univ Press, Londres, $510 p$

Cellier $P$ (1992) Mécanismes du refroidissement nocturne. Application à la prévision des gelées de prin- 
temps. In: Séminaire de la Commission d'Agrométéorologie "Le gel en agriculture», Paris, 21-22 novembre 1989 (à paraître)

Cellier P, Itier B (1984) Un modèle de prévision de la température minimale nocturne sous abri et à la surface du sol en conditions de gelées radiatives. J Rech Atmos 18, 11-22

Fritton DD, Busscher WJ, Alpert JE (1974) An inexpensive but durable thermal conductivity probe for field use. Soil Sci Soc Am Proc 38, 854-855

Fuchs M, Hadas A (1972) The heat flux density in a non-homogeneous bare loessial soil. Boundary Layer Meteorol 3, 191-200

Ghuman BS, Lal R (1985) Thermal conductivity, thermal diffusivity, and thermal capacity of some Nigerian soils. Soil Sci 139, 74-80

Hadas A (1974) Problems involved in measuring the soil thermal conductivity and diffusivity in a moist soil. Agric Meteorol 13, 105-113

Hallaire V (1988) La fissuration d'un sol argileux au cours du dessèchement. I. Description in situ. Agronomie 8, 139-145

Hopmans JW, Dane JH (1986) Thermal conductivity of two porous media as a function of water content, temperature, and density. Soil Sci 142, 187-195

Horton R, Wierenga PJ, Nielsen DR (1983) Evaluation of methods for determining the apparent thermal diffusivity of soil near the surface. Soil Sci Soc Am J 47, 25-32

Idso SB, Aase JK, Jackson RD (1975) Net radiation soil heat flux relations as influenced by soil water content variations. Boundary Layer Meteorol 9, 113-122

Jury WA, Bellantuoni B (1976) A background temperature correction for thermal conductivity probes. Soil Sci Soc Am J 40, 608-610

Laurent JP (1986) Contribution à la caractérisation des milieux poreux non granulaires : optimisation d'outils de mesure in situ des paramètres thermiques. Application à l'étude des propriétés thermi- ques du matériau terre. Thèse à l'Institut National Polytechnique de Grenoble, Grenoble, France, 151 $\mathrm{pp}+$ annexes

Mac Innes K (1981) Thermal conductivities of soils from dryland wheat regions in Eastern Washington. Thèse de Washington State University, Pullman WA, USA

Morlat R (1989) Le terroir viticole : contribution à l'étude de sa caractérisation et de son influence sur les vins. Application aux vignobles rouges de moyenne vallée de la Loire. Thèse à l'Université de Bordeaux III, Bordeaux, France, 296 pp + annexes

Al Nakshabandi G, Kohnke H (1965) Thermal conductivity and diffusivity of soils as related to moisture tension and other physical properties. Agric Meteorol 2, 271-279

Richard G, Bruckler L, Bertuzzi P (1989) Analyse critique d'une méthode d'estimation de la conductivité thermique d'un sol in situ. Soil Technol 2, 221-241

de Vries DA (1963) Thermal properties of soils. In : Physics of plant environment (WR Van Wijk, ed) North Holland Publ, Amsterdam, 210-235

de Vries DA, Peck AJ (1958a) On the cylindrical probe method of measuring thermal conductivity with special reference to soils. I. Extension of theory and discussion of probe characteristics. Aust J Phys 11, 255-271

de Vries DA, Peck AJ (1958b) On the cylindrical probe method of measuring thermal conductivity with special reference to soils. II. Analysis of moisture effects. Aust J Phys 11, 409-423

de Vries DA, Philip JR (1986) Soil heat flux, thermal conductivity and the null-alignment method. Soil Sci Soc Am J 50, 12-18

Van Wijk WR, Schneider T, Belghith A (1967) Détermination des propriétés thermiques et du flux de chaleur à la surface du sol. In: Coll Int du CNRS "Phénomènes de transport avec changement de phase dans les milieux poreux ou colloïdaux", Paris, Avril 1966, 3e séance, 125-136 